\title{
Acoplamento Magnetosfera-lonosfera sobre o setor Americano estudado por GPS
}

Alessandro J. de Abreu, Inácio M. Martin, Mauro A. Alves, Instituto Tecnológico de Aeronáutica (ITA), São José dos Campos, SP, Brasil.

Paulo R. Fagundes, Kavutarapu Venkatesh, Universidade do Vale do Paraíba (UNIVAP), São José dos Campos, SP, Brasil.

Rodolfo de Jesus, Instituto Nacional de Pesquisas Espaciais (INPE), São José dos Campos, SP, Brasil.

Mauricio Gende, Universidade Nacional de La Plata (UNLP), La Plata, Argentina.

Copyright 2016, SBGf - Sociedade Brasileira de Geofísica

Este texto foi preparado para a apresentação no VII Simpósio Brasileiro de Geofísica, Ouro Preto, 25 a 27 de outubro de 2016. Seu conteúdo foi revisado pelo Comitê Técnico do VII SimBGf, mas não necessariamente representa a opinião da SBGf ou de seus associados. É proibida a reprodução total ou parcial deste material para propósitos comerciais sem prévia autorização da SBGf.

\section{Resumo}

This study aims to investigate the magnetosphereionosphere coupling in the American sector covering from equatorial region to mid-latitudes in both hemispheres during an intense geomagnetic storm of June 2013. In this research, were analyzed observations of 10 GPS stations of the RBMC, IGS, and SAG networks through the vertical total electron content (VTEC). Observed effects include a positive phase in the northern hemisphere during the early storm and an asymmetry during the recovery phase. It has not been possible to verify phase fluctuations, concluding that this storm did not generated and even suppressed the generation of large-scale ionospheric irregularities.

\section{Introdução}

A parte ionizada da atmosfera terrestre, denominada ionosfera, é responsável por diversos fenômenos por ser altamente influenciada pelo fluxo de radiação solar na faixa do extremo ultravioleta (EUV) e raios-X. A ionosfera pode ser dividida em várias regiões ou camadas, sendo que na camada $F$ (entre aproximadamente $130 \mathrm{~km} \mathrm{e}$ $1000 \mathrm{~km}$ de altitude), podem ser observadas após o pôrdo-sol irregularidades ionosféricas. Tais irregularidades ionosféricas degradam fortemente as transmissões transionosféricas no território americano devido à grande extensão territorial (de dimensão continental) distribuída ao norte e ao sul do equador geomagnético.

As tempestades geomagnéticas são perturbações no campo geomagnético devido à entrada de partículas do vento solar na magnetosfera terrestre e são responsáveis para a geração ou supressão de irregularidades ionosféricas equatoriais (de Abreu et al., 2010a). Tempestades geomagnéticas ocorrem devido às erupções solares (solar flares) possivelmente associadas às ejeções de massa coronal (coronal mass ejection $C M E$, intensificando a velocidade e densidade do vento solar. Normalmente, uma tempestade geomagnética caracteriza-se por um início súbito (sudden storm commencement - SSC), na qual sinaliza a chegada de uma estrutura interplanetária, dando início a fase inicial. O SSC não é condição prévia para que uma tempestade geomagnética ocorra e se desenvolva. Inclui também uma fase principal e uma fase de recuperação. A fase principal é decorrente de um contínuo campo magnético interplanetário em sentido ao sul, na qual ocorre uma intensificação das partículas aprisionadas na magnetosfera que devido aos gradientes do campo geomagnético e sua curvatura, levam a deriva dessas partículas intensificando a corrente de anel. Durante esta fase, a entrada de partículas na alta latitude da atmosfera é maximizada, enquanto que na fase de recuperação, a entrada de partículas é minimizada, ou seja, a atividade geomagnética tende a normalizar-se (Gonzalez et al., 1994; Tsurutani e Gonzalez, 1997; de Abreu et al., 2014). Durante os períodos de tempestades geomagnéticas podem ocorrer modificações ionosféricas nas regiões equatoriais e de baixas latitudes. Estas modificações estão possivelmente relacionadas à penetração pontual de campos elétricos magnetosféricos e aos ventos perturbados gerados pelo aquecimento da alta atmosfera em consequência a precipitação de partículas (Abdu, 1997).

Sendo assim, o objetivo principal deste estudo consiste em investigar o acoplamento magnetosfera-ionosfera, durante uma intensa tempestade geomagnética ocorrida em junho de 2013 e consequentemente, estudar a resposta da camada $\mathrm{F}$ ionosférica em regiões equatorial e de baixas e médias latitudes no setor americano, utilizando dados do sistema de posicionamento global (GPS).

\section{Metodologia}

O sistema de posicionamento global (GPS) é composto por 24 satélites orbitando ao redor da Terra a uma altitude de aproximadamente $20.200 \mathrm{~km}$ e distribuídos em 6 órbitas com ângulo de inclinação em relação ao equador geográfico de $55^{\circ}$, transmitindo ondas eletromagnéticas nas frequências L1 (1575,42 MHz) e L2 $(1227,60 \mathrm{MHz}$ ) (Kaplan and Hegarty, 2006).

Para este estudo foram utilizados dados de GPS coletados pelas estações de Belém (BELE), Palmas (PAL), Brasília (BRAZ), Presidente Prudente (PPTE), Rio de Janeiro (RIOD) e Porto Alegre (POAL), as quais compõem a Rede Brasileira de Monitoramento Contínuo (RBMC) e disponibilizado pelo Instituto Brasileiro de Geografia e Estatística (IBGE). As estações de Bahia Blanca (VBCA) e Rio Grande (RIO2) são operadas pelo Serviço Argentino de GNSS (SAG) e as estações de Greenbelt (GODE) e St. Croix (CRO1) pertencem a rede Internacional de GNSS (IGS). As estações escolhidas no setor americano cobrem as regiões equatorial, baixas e médias latitudes (Figura 1).

Os dados de GPS foram utilizados para obter as medidas do conteúdo total de elétrons na vertical (VTEC) calculados em unidades de TEC $\left(1\right.$ TECU $=\sim 10^{16}$ elétrons $\mathrm{m}^{-2}$ ) (Hofmann-Wellenhof et al., 1994) e das flutuações de fase ou taxas de variações do TEC (ROT) calculados em (TECU $\mathrm{min}^{-1}$ ) por estação (Aarons et al., 
1996; de Abreu et al., 2010b). Os sinais GPS para medidas do VTEC e do ROT foram obtidos sob um ângulo de elevação de $20^{\circ}$.

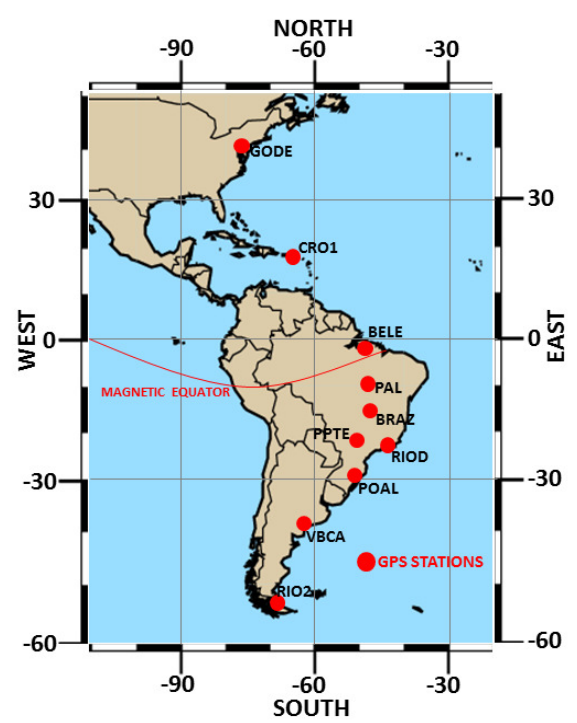

Figura 1 - Distribuição geográfica das estações de GPS.

As variações do campo geomagnético foram analisadas através dos índices $A E$ (intensidade do eletrojato auroral), $\mathrm{Kp}$ (intensidade da tempestade) e Dst (intensidade da corrente de anel). $O$ índice $A E$ é importante para obter informações relacionadas à ocorrência e intensidade de subtempestades (Sahai et al., 2007). Os dados do campo magnético interplanetário total (B), da componente vertical do campo magnético interplanetário $(\mathrm{Bz})$, da velocidade $(\mathrm{Vp})$ e densidade de prótons $(\mathrm{Np})$ do vento solar foram obtidos pelo satélite ACE $\quad$ e disponibilizados em (http://www.srl.caltech.edu/ace/).

\section{Resultados}

A Figura 2 apresenta os parâmetros do campo magnético interplanetário total $(B)$, da componente vertical do campo magnético interplanetário $(\mathrm{Bz})$, da velocidade $(\mathrm{Vp})$ e densidade de prótons $(\mathrm{Np})$ do vento solar (com resolução temporal de 64 segundos) e os índices geomagnéticos Dst (valores a cada hora), Kp (valores a cada 3 horas) e $\mathrm{AE}$. A Figura 2 inclui também, dia geomagneticamente calmo (31 de maio), dias geomagneticamente perturbados (01-03 de junho) e fases da tempestade geomagnética.

A Figura 3 apresenta o conteúdo total de elétrons na vertical (VTEC). Os valores do VTEC em faixas escuras referem-se a \pm 1 desvio padrão das médias dos dias calmos. Os valores do VTEC em linhas vermelhas referem-se aos dias que incluem, respectivamente, os dias geomagneticamente calmos e perturbados (fase principal e fase de recuperação da tempestade geomagnética). A seta e a linha tracejada vertical indica o horário em que ocorreu o sudden storm commencement (SSC).

A Figura 4 apresenta as flutuações de fase ou taxas de variações do TEC (ROT). Como foram citadas por
Wanninger (1993) e Mendillo et al. (2000), as flutuações de fase indicam a presença de irregularidades ionosféricas de grande escala ou bolhas ionosféricas (de extensão quilométrica). A Figura 4 possui o mesmo padrão de formatação da Figura 3.

\section{Discussão e Conclusões}

A Figura 2 mostra que o SSC iniciou as 00:13 UT em 01 de junho e ao mesmo tempo, o campo magnético $B$ alcançou valores acima de $20 \mathrm{nT}$. O campo Bz permaneceu para sul por mais de 03 horas, com valores ao redor de $-20 \mathrm{nT}$, enquanto a velocidade $\mathrm{Vp}$ e a densidade Np também tiveram seus valores alterados. Isso caracteriza a chegada de uma estrutura interplanetária conduzindo para a formação de uma tempestade geomagnética. Após o SSC, durante a fase principal da tempestade, o indíce Kp alcançou valores ao redor de $7+$ no período da manhã, enquanto o Dst diminuiu de aproximadamente $05 \mathrm{nT}$ para $-119 \mathrm{nT}$ em 01 de junho. Quando rápidas mudanças ocorrem no Dst durante a fase principal da tempestade, podem ocorrer penetração pontual de campos elétricos de origem magnetosférica afetando a dinâmica da ionosfera (Basu et al., 2001). Após as 09:00 UT em 01 de junho, iniciouse uma longa fase de recuperação da tempestade, se estendendo até o inicio do dia 03. Nesse período o índice $\mathrm{AE}$ mostrou fortes flutuações ao redor de $1000 \mathrm{nT}$. Isso é um indicador de grande injeção de energia em latitudes aurorais devido ao aquecimento Joule.

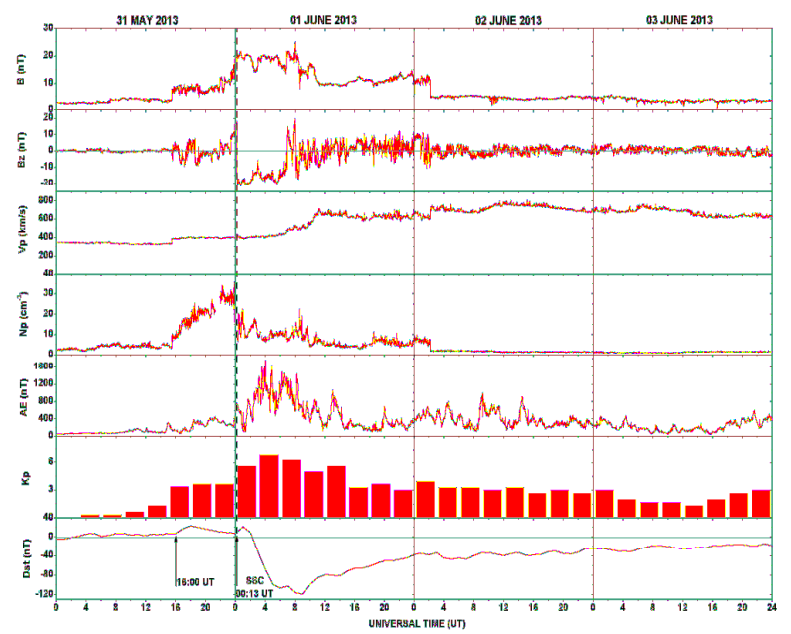

Figura 2 - Parâmetros interplanetários $B, B z, V p$ e $N p$ e as variações do campo geomagnético através dos índices $A E, K p$ e Dst entre os dias 31 de maio e 03 de junho de 2013. A seta e a linha tracejada indicam o sudden storm commencement (SSC).

A Figura 3 mostra as variações do VTEC de médias latitudes para a região equatorial em ambos os hemisférios. É possível observar em médias latitudes no hemisfério norte (GODE e CRO1) uma grande fase negativa da tempestade (diminuição do VTEC em relação ao período calmo), possivelmente devido ao impulso 
súbito ocorrido as 16:00 UT em 31 de maio e ao SSC. Fase negativa geralmente está associada a diminuição da taxa de $\mathrm{O} / \mathrm{N}_{2}$ (Danilov and Morozova, 1985). Da região equatorial para as médias latitudes no hemisfério sul (de BELE para RIO2), praticamente não tivemos variações do VTEC em relação ao período calmo. Por outro lado, durante a fase de recuperação da tempestade, é possível observar uma assimetria, onde em GODE e CRO1 podemos observar uma fase negativa e de RIO2 para BELE, uma fase positiva (aumento do VTEC em relação ao período calmo). Fase positiva está geralmente associada a mudanças na circulação de ventos (Bauske and Prolss, 1998).

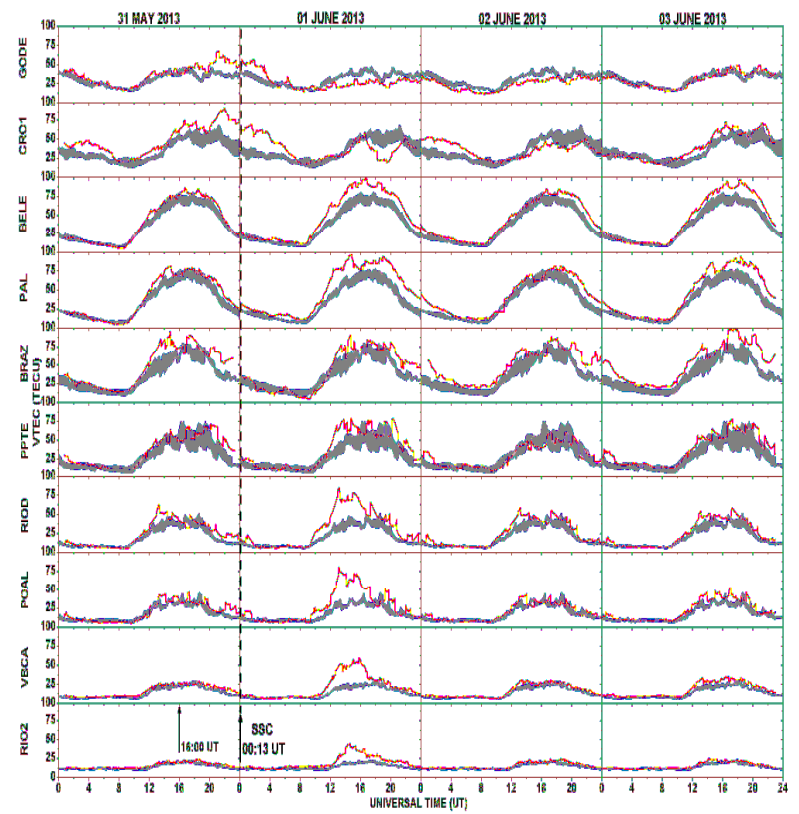

Figura 3 - Conteúdo total de elétrons na vertical (VTEC) de 10 estações de GPS obtidos entre os dias 31 de maio e 03 de junho de 2013 (linhas vermelhas) sobre o setor americano. A faixa escura corresponde a \pm 1 desvio padrão das médias dos dias calmos. A seta e a linha tracejada indicam o sudden storm commencement (SSC).

A Figura 4 mostra as flutuações de fase ou taxas de variações do TEC (ROT). É possível ver que este período, antes da tempestade e fases principal e recuperação, não foi observada irregularidades. Essa intença tempestade geomagnética não foi capaz de gerar e nem de suprimir a geração de irregularidades ionosféricas de grande escala. Isso se deve possivelmente ao foto que as flutuações de fase indicam irregularidades da ordem de quilômetros (Aarons et al., 1996).

Portanto, nesta investigação estudamos o acoplamento magnetosfera-ionosfera sobre 0 setor americano utilizando estações de GPS cobrindo de médias latitudes em ambos os hemisférios à região equatorial. Pode se concluir uma fase positiva no hemisfério norte durante 0 início da tempestade e uma assimetria durante a fase de recuperação. Não foi possível verificar flutuações de fase, concluindo que esta tempestade não gerou e nem suprimiu a geração de irregularidades ionosféricas de grande escala.

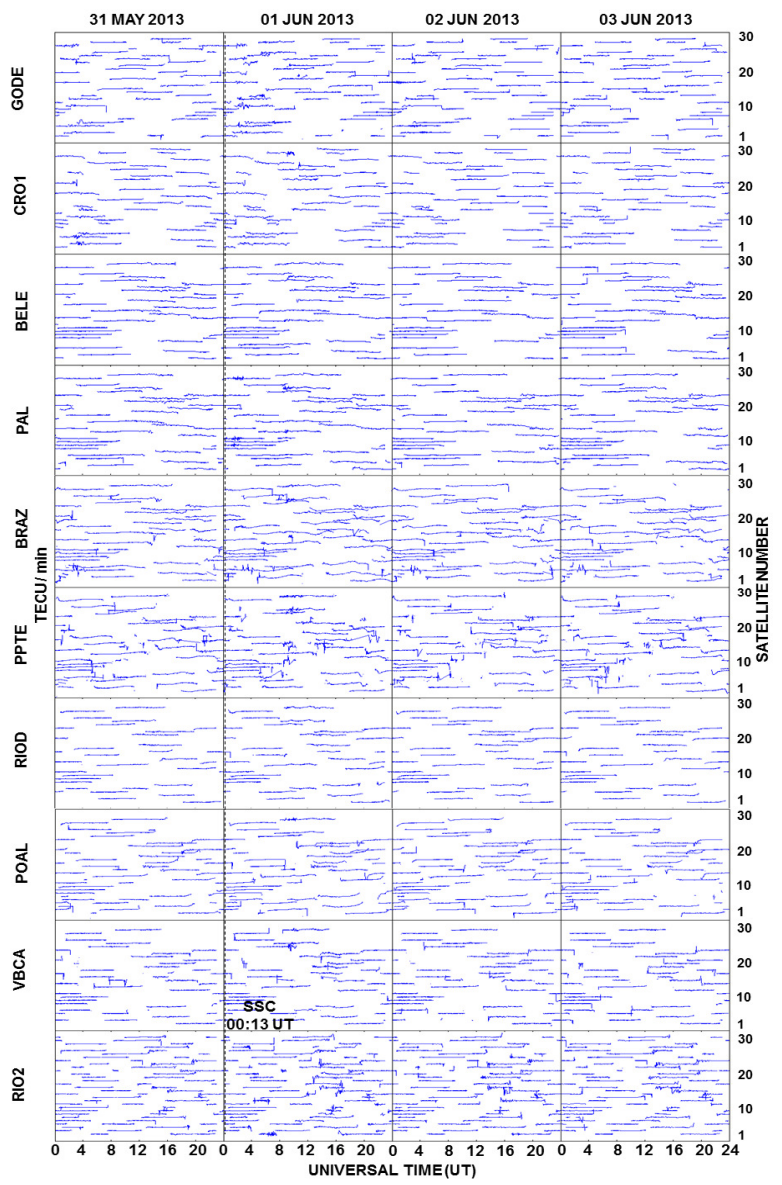

Figura 4 - Flutuações de fase ou taxas de variações do TEC (ROT) de 10 estações de GPS obtidos entre os dias 31 de maio e 03 de junho de 2013 sobre o setor americano. A linha tracejada indica o sudden storm commencement (SSC).

\section{Agradecimentos}

Os autores agradecem ao Conselho Nacional de Desenvolvimento Científico e Tecnológico (CNPq) pelo suporte financeiro (processo número 402685/2015-6), a CAPES e ao ITA pela bolsa PVS-CAPES-ITA. Também as redes RBMC, IGS e SAG pelos dados de GPS.

\section{Referências}

AARONS J, MENDILLO M \& YANTOSCA R. 1996. GPS phase fluctuations in the equatorial region during the MISETA 1994 campaign. J. Geophysical Research, v. 101, n. A8, p. $26,851-26,862$.

ABDU MA. 1997. Major phenomena of the equatorial ionosphere-thermosphere system under disturbed 
conditions. J. Atmospheric and Solar-Terrestrial Physics, v. 5 , n. 13 , p. $1505-1519$.

BASU SU, BASU SA, VALLADARES CE, YEH H-C, SU S-Y, MACKENZIE E, SULTAN PJ, AARONS J, RICH FJ, DOHERTY P, GROVES KM \& BULLET TW. 2001. Ionospheric effects of major magnetic storms during the International Space Weather Period of September and October 1999: GPS observations, VHF/UHF scintillations, and in situ density structures at middle and equatorial latitudes. J. Geophysical Research, v. 106, p. 3038930413.

BAUSKE R \& PROLSS GW. 1998. Numerical simulation of long - duration positive ionospheric storm effects. Adv. Space Res., 22(1), $117-121$.

DANILOV AD \& MOROZOVA LD. 1985. Ionospheric storms in the F2 region - Morphology and physics (review), Geomagn. Aeron., 25, 593 - 605.

de ABREU AJ, FAGUNDES PR, SAHAI Y, de JESUS R, BITTENCOURT JA, BRUNINI C, GENDE M, PILLAT VG, LIMA WLC, ABALDE JR \& Pimenta AA. 2010a. Hemispheric asymmetries in the ionospheric response observed in the American sector during an intense geomagnetic storm, Journal of Geophysical Research, v. 115, A12312.

de ABREU AJ, SAHAI Y, FAGUNDES PR, BECKERGUEDES F, de JESUS R, GUARNIERI FL \& Pillat VG. $2010 \mathrm{~b}$. Response of the ionospheric F-region in the Brazilian sector during the super geomagnetic storm in April 2000 observed by GPS. Advances in Space Research, v. 45, p. 1322-1329.

de ABREU AJ, FAGUNDES PR, GENDE M, BOLAJI OS, de JESUS R \& BRUNINI C. 2014. Investigation of ionospheric response to two moderate geomagnetic storms using GPS-TEC measurements in the South American and African sectors during the ascending phase of solar cycle 24. Advances in Space Research, v. 53, p. 1313-1328.
GONZALEZ WD, JOSELYN JA, KAMIDE Y, KROEHL HW, ROSTOKER G, TSURUTANI BT \& VASYLIUNAS VM. 1994. What is a magnetic storm? J. Geophysical Research, v. 99, n. A4, p. 5771-5792.

HOFMANN-WELLENHOF B, LICHTENEGGER H \& COLLINS J. 1994. Global Positioning System: Theory and Practice. 3. ed. Wien: Springer-Verlag.

KAPLAN ED \& HEGARTY CJ. 2006. Understanding GPS: Principles and Applications. 2. ed. Artech House, Inc.

MENDILLO M, LIN B \& AARONS J. 2000. The application of GPS observations to equatorial aeronomy. Radio Science, v. 35, n. 3, p. 885-904.

SAHAI Y, BECKER-GUEDES F, FAGUNDES PR, LIMA WLC, OTSUKA Y, HUANG C-S, ESPINOZA ES, PI X, DE ABREU AJ, BOLZAN MJA, PILLAT VG, ABALDE JR, PIMENTA AA \& BITTENCOURT JA. 2007. Response of nighttime equatorial and low latitude F-region to the geomagnetic storm of August 18, 2003, in the Brazilian sector. Advances in Space Research, n. 39, p. 13251334.

TSURUTANI BT \& GONZALEZ WD. 1997. The interplanetary cause of magnetic storms: a review. In: Tsurutani, B. T; Gonzalez, W. D; Kamide, Y; Arballo, J. K. ed. Magnetic storms. Washington, DC, v. 98.

WANNINGER L. 1993. Effects of the Equatorial Ionosphere on GPS. GPS World, p. 48-54, July. 